\title{
Preface
}

\section{The Evolving Role of \\ Molecular Diagnostics in Pathology}

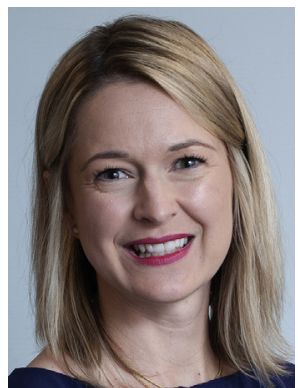

Lauren L. Ritterhouse, MD, PhD

Editor

The clinical implementation of molecular diagnostic technologies continues to expand across a wide variety of tumor types and has been shown to have utility for diagnostic, prognostic, and therapeutic implications. Genomic studies continue to expand our understanding of pathobiology with revelations that some differing tumor types are part of the same spectrum of disease; such is the case with esophageal adenocarcinoma and intestinal-type gastric adenocarcinomas of the proximal stomach (Stachler and Jin). Novel biomarkers are rapidly and continuously being identified, necessitating frequent workflow and best practice optimizations in both surgical pathology and the molecular diagnostic laboratory to facilitate the implementation of guideline recommended biomarker testing. In addition, there is an influx of new technologies and diagnostic assays available, such that curation of a molecular diagnostic pathology test menu for both in-house and send-out testing requires significant thought and collaboration with clinical colleagues (Hsaio and Ewalt). Depending on the tumor type and biomarkers to be evaluated, screening with immunohistochemistry may play an important role (Hernandez-Prera).

In addition, as many new biomarkers and targeted therapies have demonstrated evidence and efficacy across tumor types, the field of pantumor biomarkers continues to evolve (Dong). Prior to 2017, there were no Food and Drug Administration approvals for cancer therapies based exclusively on the presence of a biomarker and not tissue of origin. Since then, pembrolizumab has been approved for microsatelliteinstability-high or mismatch repair deficient solid tumors in 2017, larotrectinib for solid tumors with NTRK gene fusions in 2018, and entrectinib for solid tumors with NTRK gene fusions in 2019. Although PARP inhibitors do not have a pantumor approval, several biomarkers, such as BRCA1/2 mutations and homologous recombination deficiency, may predict response to PARP inhibition across several tumor types, such as ovarian (Ordulu, Watkins, Ritterhouse) and prostate cancer (Kulac, Roudier, Haffner). Additional genes and signatures involved in DNA damage response and repair are also being evaluated as biomarkers predicting response to both chemo0therapies and immunotherapies, exemplified in the case of urothelial carcinoma (Al-Ahmadie and Netto).

World Health Organization classifications for both central nervous system tumors and myeloid neoplasms have incorporated molecular and cytogenetic features into their classification criteria (Galbraith and Snuderl; Sadigh and Kim), and are expected to continue to evolve. New diagnostic entities are emerging in both sarcomas and pediatric solid tumors (Slack and Church) that are defined by their genetics, such as RET- and NTRK-rearranged mesenchymal tumors, which may be identical morphologically but respond to unique targeted therapies. 
Frequent referencing of guideline statements, such as the National Comprehensive Cancer Network clinical practice guidelines in oncology, is highly recommended due to the constantly evolving nature of biomarkers. This is particularly true in tumor types such as non-small cell lung cancer in which genotyping is the standard of care to guide optimal therapy selection (RoyChowdhuri). In addition to genotyping at diagnosis, the role of molecular testing at recurrence or disease progression is growing as we learn more about resistance mechanisms to various therapies (Roy-Chowdhuri; Ross and Pareja). The utilization of so-called liquid biopsies (plasmabased circulating tumor DNA evaluation) continues to evolve, with potential applications in tumor heterogeneity, tissue inadequacy, and for emerging roles, such as predicting tumor recurrence in colorectal cancer (Beech and Hechtman). Finally, indications for measurable residual disease molecular testing are best established in hematopoietic neoplasms (Xian and Ware; Sadigh and Kim). As is evidenced in the following articles, molecular diagnostics is a promising, rapidly evolving, and integral part of the modern pathology practice.

Lauren L. Ritterhouse, MD, PhD Center for Integrated Diagnostics

Department of Pathology Massachusetts General Hospital Harvard Medical School Jackson Building 10th Floor, Suite 1015 55 Fruit Street Boston, MA 02114, USA

E-mail address: Iritterhouse@mgh.harvard.edu 